\title{
Unsafe abortion: a cruel way of birth control
}

\author{
Saurabh RamBihariLal Shrivastava, Prateek Saurabh Shrivastava, Jegadeesh Ramasamy
}

1. Department of Community Medicine, Shri Sathya Sai Medical College \& Research Institute, Kancheepuram.

\begin{abstract}
Background: Unsafe abortion refers to a procedure for terminating an unintended pregnancy performed either by persons lacking the necessary skills or in an environment that does not conform to minimal medical standards, or both.

Objectives: The objectives of the study are to assess the factors attributing to practice of unsafe abortion and to suggest feasible and cost-effective measures to counter the same.

Methods: An extensive search of all materials related to the topic was made using library sources including Pubmed, Medline and World Health Organization. Keywords used in the search include unsafe abortion and unintended pregnancy.

Results: Multiple socio-demographic determinants and barriers such as illiterate women, poor socio-economic status, poor awareness about abortion services, associated stigma, and untrained health professionals have been identified resulting in restricted utilization/access of women to safe abortion services. Consequences of unsafe abortion have been alarming, seriously questioning the quality of health care delivery system.

Conclusion: Concerted and dedicated efforts of government in collaboration with the private sector, community members and non-governmental organizations are needed to ensure that women have a better access to contraceptives, abortion services, and post-abortion care that are safe, affordable, and free from stigma.
\end{abstract}

Keywords: Unsafe abortion, unintended pregnancy, Millennium development goals, Public health

African Health Sciences 2014; 14(2):487-488

DOI: http://dx.doi.org/10.4314/ahs.v14i2.29

Sir,

Unsafe abortion refers to a procedure for terminating an unintended pregnancy performed either by persons lacking the necessary skills or in an environment that does not conform to minimal medical standards, or both. ${ }^{1}$ Although definition refers to the process, unsafe abortion actually reflects unsuitable situations before, during or after an abortion such as lack of contraception, no pre-abortion advice; unhygienic conditions; induced

\section{Corresponding author:}

Saurabh RamBihariLal Shrivastava

Address: Department of Community

Medicine,Shri Sathya Sai Medical College

$\&$ Research Institute, Ammapettai village,

Thiruporur - Guduvancherry Main Road, Sembakkam Post, Kancheepuram - 603108, Tamil Nadu, India

Telephone: +919884227224

E-mail: drshrishri2008@gmail.com by an unskilled provider or by insertion of an object into the uterus or by medication with no follow-up, etc. ${ }^{1,2}$ Globally in 2008, 21.6 million women underwent an unsafe abortion, of which 18.5 million alone were from developing nations, with the highest contributions from Asian (10.8 million) and African (6.2 million) regions. ${ }^{2}$ Despite global attempts to attain Millennium Development Goal 5A - unsafe abortion contribution to maternal mortality has remained constant at $13 \%{ }^{2}$ Multiple socio-demographic determinants and barriers such as young age $;^{3}$ illiterate women; ${ }^{3}$ socio-cultural and religious beliefs; ${ }^{4}$ poor socio-economic status; ${ }^{3}$ poor awareness about abortion services; ${ }^{4}$ health center related factors - geographical inequities / inadequate number / poor quality; ${ }^{2,3}$ associated stigma; ${ }^{4,5}$ legislative restrictions, ${ }^{2,5}$ unwillingness among policy-makers to implement abortion laws; ${ }^{3}$ untrained health professionals; ${ }^{2,5}$ monetary constraints in affording safe abortion; ${ }^{2}$ and attitude of health staff towards clients; ${ }^{2}$ have been identified resulting in restricted utilization/ access of women to safe abortion services.

Consequences of unsafe abortion have been alarming, 
seriously questioning the quality of healthcare delivery system of the country. As per recent estimates, annually 47000 women die from complications originating from unsafe abortion in the year 2008 alone. $^{2}$ In addition, it has been accounted as a significant cause of ill-health among women; ${ }^{1}$ increasing the rate of hospitalization; ${ }^{6}$ hampering women's ability to conceive in future, ${ }^{3}$ infertility; ${ }^{3}$ finally increasing the direct and indirect involved medical cost; ${ }^{7}$ and over-burdening of the health system. ${ }^{7}$

Concrete customized measures must be formulated and implemented for minimizing the incidence of unsafe abortion and its associated complications in developing nations and in low-resource settings. The first priority is to improve the quality of data collection, so that appropriate strategies can be planned and rational allocation of resources done. Measures such as - improvement in community awareness about the availability of safe abortion services and the eligibility criteria for undergoing abortion legally under the country's acts / regulations; ' networking with all stakeholders belonging to different political and social structures; ${ }^{2}$ infrastructure strengthening; ${ }^{5}$ capacity building - ensuring healthcare staff training in safe abortion practices;, ${ }^{5,9}$ enhancing accessibility to safe abortion services; ${ }^{2,6,9}$ community participation; ${ }^{2,5}$ securing adequate contraceptive supplies; $;, 10$ measures for adolescent girls - preventing early marriage/ delaying early pregnancy through sex education/ facilitating access to welfare programs/ promoting adoption of family spacing methods; $;, 10$ minimizing unintended pregnancy ${ }^{10}$ increasing partner and family support; ${ }^{4}$ advocating change in behavior of healthcare providers; ${ }^{2,11}$ and empowering women to decide whether and when to have a child; ${ }^{2,11}$ deserves significant importance in the current era to counter the problem of unsafe abortions.

To conclude, concerted and dedicated efforts of government in collaboration with the private sector, community members and non-governmental organizations are needed to ensure that women have a better access to contraceptives, abortion services, and post-abortion care that are safe, affordable, and free from stigma.

\section{Source of support:}

None

\section{Conflicts of interests:}

None to be declared.

\section{References}

1. World Health Organization. The prevention and management of unsafe abortion. WHO Press: Geneva, 1992.

2. World Health Organization. Unsafe abortion - Global and regional estimates of the incidence of unsafe abortion and associated mortality in 2008. WHO Press: Geneva, 2011.

3. Machado CJ, Lobato AC, Melo VH, Guimaraes MD. Spontaneous and voluntary fetal losses in Brazil in 1999-2000: a study of associated factors. Rev Bras Epidemiol 2013; 16(1): 18-29.

4. Harris LH. Stigma and abortion complications in the United States. Obstet Gynecol 2012; 120(6): 1472-1474.

5. Culwell KR, Hurwitz M. Addressing barriers to safe abortion. Int J Gynaecol Obstet 2013; 121(Suppl 1): S1619.

6. Prada E, Singh S, Villarreal C. Health consequences of unsafe abortion in Colombia, 1989-2008. Int J Gynaecol Obstet 2012; 118(Suppl 2): S92-98.

7. Babigumira JB, Stergachis A, Veenstra DL, et al. Estimating the costs of induced abortion in Uganda: a model-based analysis. BMC Public Health 2011; 11: 904.

8. Gerdts C, Vohra D, Ahern J. Measuring unsafe abortion-related mortality: a systematic review of the existing methods. PLoS One 2013; 8(1): e53346.

9. Banerjee SK, Andersen K. Exploring the pathways of unsafe abortion in Madhya Pradesh, India. Glob Public Health 2012; 7(8): 882-896.

10. Chandra-Mouli V, Camacho AV, Michaud PA. WHO guidelines on preventing early pregnancy and poor reproductive outcomes among adolescents in developing countries. J Adolesc Health 2013; 52(5): 517-522.

Hossain A, Maddow-Zimet I, Singh S, Remez L. Menstrual regulation, unsafe abortion, and maternal health in Bangladesh. Issues Brief (Alan Guttmacher Inst). 2012; 3: $1-8$. 